\title{
DNA Constructs
}

nlp-12 Entry Vectors (OMF761) 5'-CACCATTATTTTTTAAATTTCTATTTTTTTC-3'

(forward) and (OMF762) 5'-TTGCCATTCCCAGATTGGCTCGAGC-3' or (OMF860) 5'CACCTTTGTCGGAGGCAATTGAAATAAGTTT-3' were utilized for generating the nlp12 entry clones. CACC was added to the 5 ' end of primers for directional cloning. The reverse primers were designed either just adjacent to the start codon ( $n / p-12$ promoter) or at the end of the coding region excluding the stop (nlp-12 genomic).

Destination Vectors All destination vectors were engineered to contain the $c c d B$ reading frame $\mathrm{B}$ cassette (Invitrogen, CA).

pDest-17: $c c d B$ cassette was Smal/Sphl subcloned into an existing plasmid containing mCherry to generate the destination vector pDest-17 (mCherry).

pDest-27: $c c d B$ cassette was Mfel/Sphl subcloned into an existing plasmid (pDest-24)

containing the trans-splice leader and acceptor sequences followed by mCherry to obtain pDest-27 (SL2::mCherry).

pDest-25 and pDest-34: 720 bp VenusYFP and 1374 bp Tetanus light chain fragments were PCR amplified with 5'-ATTAACCGGTATGGTGAGCAAGGGCGAGGA-3' (OMF753), 5'GCTAGCCGGCTTACTTGTACAGCTCGTCCA-3' (OMF754) and 5'-ATTAACCGGTATGCCGATCACCATCAACAAC-3' (OMF883), 5'-GCTAGCCGGCTTAAGCGGTACGGTTGTACA-3' (OMF884) primer pairs. The forward and reverse primers contained restriction sites (underlined) for Agel and NgoMIV, respectively. Digested PCR products were subcloned into Agel/NgoMIV sites of an existing destination vector (pDest-8) replacing mCherry, to generate pDest-25 (VenusYFP) and pDest-34 (Tetanus toxin light chain).

pDest-60: pCL33 (pacr-2::dop-1) [51] was digested with BstZ171 and Nhel to remove the acr-2 promoter fragment. $c c d B$ cassette was cloned in to generate pDest-60 (dop-1). 